\title{
Dried Blood Spot Multiplexed Steroid Profiling Using Liquid Chromatography Tandem Mass Spectrometry in Korean Neonates
}

\author{
Rihwa Choi, M.D. 1,2,3, Hyung-Doo Park (ש), M.D., Ph.D. ${ }^{2,3}$, Hyeon Ju Oh, M.S. ${ }^{2}$, Kyounghoon Lee, M.D. ${ }^{2,3}$, \\ Junghan Song, M.D., Ph.D. ${ }^{4}$, and Soo-Youn Lee (0), M.D., Ph.D. ${ }^{2,3}$ \\ ${ }^{1}$ Department of Laboratory Medicine, Green Cross Laboratories, Yongin, Korea; ${ }^{2}$ Department of Laboratory Medicine and Genetics, Samsung Medical Center, \\ Seoul, Korea; ${ }^{3}$ Department of Laboratory Medicine and Genetics, Sungkyunkwan University School of Medicine, Seoul, Korea; ${ }^{4}$ Department of Laboratory \\ Medicine, Seoul National University College of Medicine, Seoul National University Bundang Hospital, Seongnam, Korea
}

Background: Screening for congenital adrenal hyperplasia (CAH) using immunoassays for $17 \alpha$-hydroxyprogesterone generates many false-positive results. We developed and validated a liquid chromatography-tandem mass spectrometry (LC-MS/MS) assay for simultaneous quantification of nine steroid hormones in dried blood spot (DBS) samples, and established reference intervals for these hormones.

Methods: We examined our method for linearity, precision, accuracy, extraction recovery, and matrix effects and determined the reference intervals of cortisol, 17 $\alpha$-hydroxyprogesterone, 11-deoxycortisol, 21-deoxycortisol, androstenedione, corticosterone, 11-deoxycorticosterone, testosterone, and progesterone in 1,146 DBS samples (from 272 preterm and 874 full-term neonates). Immunoassay and LC-MS/MS methods were compared for $17 \alpha$-hydroxyprogesterone. Fourteen additional samples were tested to validate the clinical applicability of the LC-MS/MS method.

Results: The linearity range was $2.8-828.0 \mathrm{nmol} / \mathrm{L}$ for cortisol and $0.9-40.0 \mathrm{nmol} / \mathrm{L}$ for the other steroids $\left(R^{2}>0.99\right)$. Intra-day and inter-day precision CVs were $2.52-12.26 \%$ and $3.53-17.12 \%$, respectively. Accuracy was $80.81-99.94 \%$, and extraction recovery and matrix effects were $88.0-125.4 \%$ and $61.7-74.2 \%$, respectively. There was a negative bias, with higher values measured by immunoassay compared with LC-MS/MS $(r=0.8104, P<$ 0.0001). The LC-MS/MS method was successfully applied to the analysis of nine steroids in DBS for screening and diagnosis of CAH using the 14 additional samples.

Conclusions: Our method enables highly sensitive and specific assessment of nine steroids from DBS and is a promising tool for clinical analysis of $\mathrm{CAH}$.

Key Words: $17 \alpha$-hydroxyprogesterone, Steroid hormones, Congenital adrenal hyperplasia, reference intervals, LC-MS/MS, Dried blood spot
Received: June 10, 2018

Revision received: September 7, 2018

Accepted: December 17, 2018

\section{Corresponding author: \\ Hyung-Doo Park, M.D. \\ (iD https://orcid.org/0000-0003-1798-773X Department of Laboratory Medicine and Genetics, Samsung Medical Center, Sungkyunkwan University School of Medicine, 81 Irwon-ro, Gangnam-gu, Seoul 06351, Korea \\ Tel: +82-2-3410-0290 \\ Fax: +82-2-3410-2719 \\ E-mail: nayadoo@hanmail.net}

\section{Co-corresponding author:}

Soo-Youn Lee, M.D.

(iD https://orcid.org/0000-0001-7595-4042

Department of Laboratory Medicine and

Genetics, Samsung Medical Center,

Sungkyunkwan University School of

Medicine, 81 Irwon-ro, Gangnam-gu, Seoul 06351, Korea

Tel: +82-2-3410-1834

Fax: +82-2-3410-2719

E-mail: sy117.lee@samsung.com

\section{(C) Korean Society for Laboratory Medicine \\ This is an Open Access article distributed under the terms of the Creative Commons Attribution Non-Commercial License (http://creativecom- mons.org/licenses/by-nc/4.0) which permits unrestricted non-commercial use, distribution, and reproduction in any medium, provided the original work is properly cited.}

\section{INTRODUCTION}

Serum steroid assays play an important role in the clinical evaluation of many common endocrine disorders [1]. Congenital adrenal hyperplasia $(\mathrm{CAH})$, the most common adrenal gland disorder in infants and children, is a group of autosomal recessive disorders [2]. The 21-hydroxylase catalyzes hydroxylation of 17 $\alpha$-hydroxyprogesterone to 11-deoxycortisol in the glucocorticoid pathway [1]. In particular, 21-hydroxylase deficiency is the cause of approximately $95 \%$ of CAH cases [2]. Most neonatal screening pro- 
grams worldwide include the detection of $\mathrm{CAH}$, and the standard test parameter is $17 \alpha$-hydroxyprogesterone $[3,4]$.

Although immunoassays to evaluate $17 \alpha$-hydroxyprogesterone are easy to handle, rapid, and highly sensitive, they have questionable reliability because of a lack of specificity to $\mathrm{CAH}$ and matrix effects [5]. Conventional screening for $\mathrm{CAH}$ using immunoassays for $17 \alpha$-hydroxyprogesterone generates many falsepositive results [3,5], which not only upset parents and medical staff but also necessitate subsequent costly clinical and laboratory analyses [3].

While the inclusion of extraction steps improves immunoassay specificity, all interfering molecules cannot be completely eliminated [5]. Several studies attribute the low specificity to crossreactivity of antibodies with steroids other than $17 \alpha$-hydroxyprogesterone, such as steroid sulfates, $17 \alpha$-hydroxypregnenolone, and $17 \alpha$-hydroxypregnenolone sulfate $[1,3,5]$. Poor immunoassay specificity is an issue, especially in preterm or stressed infants, who have high concentrations of delta- 5 cross-reacting steroids [2]. Preterm neonates often have high concentrations of $17 \alpha$-hydroxyprogesterone because of stress or delayed maturation of 11-hydroxylase [1], which can reduce diagnostic specificity in screening for CAH by antibody-based methods, potentially resulting in false-positive diagnosis and generating demand for a second-tier confirmation test [2]. Using steroid profiling by liquid chromatography-tandem mass spectrometry (LC-MS/MS) in neonate screening for $\mathrm{CAH}$ yields fewer false-positive results because of its high analytical specificity and potential to quantify several compounds in a single run [2].

LC-MS/MS is the most accurate method currently available for measuring small molecules, and it can measure multiple steroid hormones simultaneously [6]. Steroid profiling with LCMS/MS allows for evaluation of the status of enzymes involved in adrenal steroid biosynthetic pathways; thus, it is a better diagnostic tool than the evaluation of a single steroid [6]. A specific LC-MS/MS method with simultaneous detection of multiple steroids has been introduced to minimize unnecessary followup analyses [3, 6]. Although previous studies examined multiple steroid hormonal analyses using LC-MS/MS in Korea, they included limited numbers of steroids [6, 7] or did not evaluate clinical screening for $\mathrm{CAH}$ using patient samples [6]. Testosterone and progesterone, steroid hormones useful for the screening and diagnosis of $\mathrm{CAH}$, have not been studied using dried blood spots (DBS) in Korean neonates [6, 7].

We developed and validated an LC-MS/MS method that accurately detects cortisol, 17 $\alpha$-hydroxyprogesterone, 11-deoxycortisol, 21-deoxycortisol, androstenedione, corticosterone, 11-deoxycorticosterone, testosterone, and progesterone in DBS, so as to be suitable for the screening and diagnosis of $\mathrm{CAH}$ in neonates. As the reference interval is not only method-specific [8] but also age-specific [9], we also determined age-specific reference intervals for the nine aforementioned steroids for healthy Korean neonates, including preterm neonates.

\section{METHODS}

\section{Reagents, instruments, analytical conditions, and sample preparation}

Samples were collected on DBS cards (Honeywell Burdick \& Jackson, Morristown, NJ, USA) between May 2013 and November 2016 at Samsung Medical Center, Seoul, Korea. Reagents, instruments, analytical conditions, and sample preparation were as described previously $[6,8]$ with modifications for two more steroid hormones (testosterone and progesterone). Quantitative analyses were conducted in multiple reaction monitoring mode using an Agilent 6490 Triple Quadrupole Mass Spectrometer equipped with an Agilent 1260 Infinity HPLC system (Agilent Technologies, Santa Clara, CA, USA).

Operating conditions are shown in Table 1. To determine reference intervals for the nine steroids in Korean subjects, the 1,146 DBS samples from neonates (272 from preterm and 874 from full-term neonates) were analyzed using the LC-MS/MS method, and the data were stratified by sex.

\section{Assay performance characteristics}

Assay performance characteristics, including assay range, lower limit of quantification (LLOQ), linearity, precision, accuracy, extraction recovery, and matrix effects were evaluated according to the updated guidelines and literature for MS/MS method validation and neonate screening [2, 10-27]. Accuracy was additionally assessed by participating in the second-tier Congenital Adrenal Hyperplasia Proficiency Testing Program (CAHPT), a proficiency test for immunoassay and LC-MS/MS methods published quarterly by the Newborn Screening Quality Assurance Program of the Centers for Disease Control and Prevention [4]. In total, 1,146 anonymized DBS samples submitted for routine clinical testing were used to compare $17 \alpha$-hydroxyprogesterone concentrations determined by immunoassay (AutoDELFIA; PerkinEImer, Waltham, MA, USA) and LC-MS/MS.

\section{Reference intervals}

Reference intervals were determined according to the CLSI guidelines [9] and compared with previously reported intervals 
Table 1. HPLC gradient conditions and mass spectrometer operating conditions

\begin{tabular}{|c|c|c|c|c|c|c|}
\hline \multicolumn{7}{|c|}{ HPLC gradient condition } \\
\hline Time (min) & Mobile A (\%) & Mobile B (\%) & & & & \\
\hline 0 & 90 & 10 & & & & \\
\hline 4 & 58 & 42 & & & & \\
\hline 8 & 51 & 49 & & & & \\
\hline 10.5 & 34 & 66 & & & & \\
\hline 11.5 & 10 & 90 & & & & \\
\hline 13 & 10 & 90 & & & & \\
\hline 13.5 & 90 & 10 & & & & \\
\hline \multicolumn{7}{|c|}{ Mass spectrometer operating conditions } \\
\hline Time segment & Compound name & $\mathrm{RT}(\min )$ & Dwell time (msec) & Precursor- & t ion $(\mathrm{m} / \mathrm{z})$ & $\mathrm{CE}(\mathrm{eV})$ \\
\hline \multirow[t]{2}{*}{1} & Cortisol & 5.903 & 200 & 363.1 & 121 & 30 \\
\hline & d4-Cortisol & 5.879 & 200 & 367.4 & 121.1 & 22 \\
\hline \multirow[t]{6}{*}{2} & 21-Deoxycortisol & 6.751 & 100 & 347.2 & 311 & 16 \\
\hline & d8-21-Deoxycortisol & 6.683 & 100 & 355.3 & 100 & 32 \\
\hline & Corticosterone & 7.084 & 100 & 347.2 & 121 & 22 \\
\hline & d8-Corticosterone & 6.987 & 100 & 355.2 & 337.1 & 14 \\
\hline & 11-Deoxycortisol & 7.438 & 100 & 347.2 & 109 & 28 \\
\hline & d2-11-Deoxycortisol & 7.405 & 100 & 349.3 & 109 & 34 \\
\hline \multirow[t]{8}{*}{3} & Androstenedione & 8.281 & 50 & 287.2 & 97 & 16 \\
\hline & d7-Androstendione & 8.201 & 50 & 294.2 & 100 & 22 \\
\hline & Testosterone & 9.043 & 50 & 289.2 & 109.1 & 22 \\
\hline & d5-Testosterone & 8.916 & 50 & 294.2 & 99.9 & 22 \\
\hline & 11-Deoxycorticosterone & 9.200 & 500 & 331.3 & 97.1 & 32 \\
\hline & d8-11-Deoxycorticosterone & 9.115 & 50 & 339.5 & 100 & 27 \\
\hline & $17 \alpha$-Hydroxyprogesterone & 9.775 & 50 & 331.3 & 97.2 & 32 \\
\hline & d8-17 $\alpha$-Hydroxyprogesterone & 9.697 & 50 & 339.5 & 100.1 & 27 \\
\hline \multirow[t]{2}{*}{4} & Progesterone & 11.196 & 100 & 315.2 & 109.2 & 30 \\
\hline & d9-Progesterone & 11.133 & 100 & 324.2 & 113.1 & 26 \\
\hline
\end{tabular}

Abbreviations: RT, retention time; CE, collision energy.

in the other populations $[6,8,23-25,27]$. To validate the reference intervals, we used additional anonymized samples from eight neonates without CAH (normal neonates), three full-term neonates with CAH (21-hydroxylase deficiency) whose 17 $\alpha$-hydroxyprogesterone was $>18.2 \mathrm{nmol} / \mathrm{L}$ by immunoassay, two girls (4 and 14 years old) with CAH (21-hydroxylase deficiency), and a 3-year-old girl with congenital adrenal lipoid hyperplasia due to StAR mutation.

\section{Statistical analyses}

All data for steroid hormones with or without stratification by age and sex were not normally distributed. We used the Mann-Whitney $\mathrm{U}$ test to compare them. Reference inervals were estimated as the central 95\% (the 2.5th and 97.5th percentiles) of the distribution of test results according to the CLSI guidelines [9]. $P<0.05$ was considered significant. Bland-Altman plots were created, and the nonparametric Passing-Bablock regression was conducted to compare the immunoassay and LC-MS/MS for 17 $\alpha$-hydroxyprogesterone, using MedCalc software for Windows, version 17.9.7 (MedCalc Software, Ostend, Belgium) [17].

\section{Ethics}

This study was conducted according to the Declaration of Helsinki, and all procedures involving human subjects were approved by the Institutional Review Board of Samsung Medical Center (SMC 2012-08-058), Seoul, Korea. 


\section{RESULTS}

\section{Performance of LC-MS/MS for nine steroid hormones}

Analyses of steroid-free samples showed no interfering peaks at either steroid or internal standard retention times. The total run time for each sample was 16 minutes. There were linear correlations between steroid concentration and signal intensity for cortisol (2.8-828.0 nmol/L) as well as for the other steroids (0.9$40.0 \mathrm{nmol} / \mathrm{L} ; \mathrm{R}^{2}>0.99$ ). In addition, steroid concentrations that were 10 times higher than the highest calibrator still complied with linearity [14, 24]. Intra-day precision and inter-day precision CVs were $2.52-11.54 \%$ and $3.53-17.12 \%$, respectively. Accuracy was 80.81-99.94\% (difference: 0.06-19.19\%). High accuracy was assured by participating in CAHPT and having acceptable results for four quarters [4, 14]. Extraction recovery (the average percent of the target concentration recovered from DBS calibrators run as patient samples) was $64.3-74.2 \%$, and the matrix effect was 88.0-125.4\%.

In the comparison between the LC-MS/MS method and immunoassay for $17 \alpha$-hydroxyprogesterone, the LLOQ of the immunoassay for $17 \alpha$-hydroxyprogesterone determination was 0.3 $\mathrm{nmol} / \mathrm{L}$. The correlation coefficient $(r)$ was $0.8104(95 \% \mathrm{Cl}$ : $0.7896-0.8294, P<0.0001$ ), and there was a negative bias, with higher values measured by immunoassay than by LC-MS/ MS over the entire data range (Fig. 1).

Reference intervals and clinical application to 21-hydroxylase deficiency

Reference intervals of all steroid hormones except testosterone and progesterone significantly differed between preterm and full-term neonates (Table 2, $P<0.0001$ ). Reference intervals and median values of the nine steroids determined in this study in comparison with those reported by other studies are presented in Tables 3 and 4 . Of the nine steroids, only testosterone concentrations differed significantly by sex. The LC-MS/MS method allowed reliable differentiation of samples from 21-hydroxylase-deficient and unaffected neonates (Fig. 2). Of the two children with 21-hydroxylase deficiency, the 14-year-old girl was identified as screen positive for 21-hydroxylase deficiency with increased 17 $\alpha$-hydroxyprogesterone, 21-deoxycortisol, and androstenedione, and the 4-year-old girl was screen positive with only increased 17 $\alpha$-hydroxyprogesterone (her cortisol concentration was higher than the upper limit of the reference interval for full-term neonates). The ratio of androstenedione+17 $\alpha$-hydroxyprogesterone to cortisol of the children was not higher than the upper limit of the reference interval for either full-term neonates or preterm neonates. For the 3-year-old girl with congenital adrenal lipoid hyperplasia caused by StAR mutation, the cortisol concentration was higher than the upper limit of the reference interval for full-term neonates, whereas the other steroids were within the reference interval for full-term neonates.

\section{DISCUSSION}

We developed and validated an LC-MS/MS method for the simultaneous quantification of nine steroids in DBS. In addition, we determined age-specific reference intervals of the nine steroids for Korean neonates (preterm and full-term). To the best of our knowledge, this is the first attempt to determine reference intervals for testosterone and progesterone concentrations in
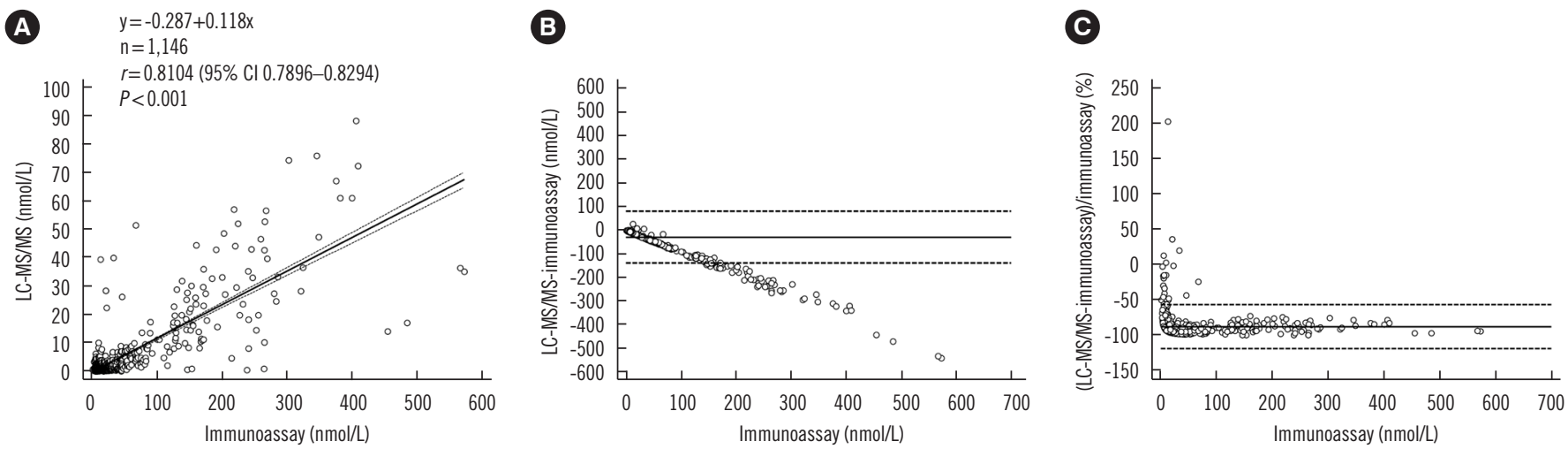

Fig. 1. Comparison of LC-MS/MS and immunoassay. Correlation (A), absolute difference (B), and percent bias (C) between LC-MS/MS and immunoassay results for $17 \alpha$-hydroxyprogesterone in 1,146 dried blood spots from Korean neonates. The solid line in (A) is the best-fit regression line and dashed lines in (A) represent 95\% confidence interval. Solid lines in (B) and (C) represent average differences, and dashed lines in (B) and (C) represent limit of agreement.

Abbreviation: LC-MS/MS, liquid chromatography-tandem mass spectrometry. 
Table 2. Reference intervals (nmol/L) for nine steroid hormones in dried blood spots from Korean preterm and full-term neonates

\begin{tabular}{|c|c|c|c|c|c|c|c|}
\hline & \multicolumn{3}{|c|}{ Preterm neonates } & \multicolumn{3}{|c|}{ Full-term neonates } & \multirow[b]{2}{*}{$P$} \\
\hline & $\mathrm{N}$ & Median & $\begin{array}{l}\text { Central 95th } \\
\text { percentile }\end{array}$ & $\mathrm{N}$ & Median & $\begin{array}{l}\text { Central 95th } \\
\text { percentile }\end{array}$ & \\
\hline Cortisol & 272 & 29.31 & $<2.76-1,026.66$ & 874 & 18.77 & $<2.76-188.88$ & 0.0001 \\
\hline 21-Deoxycortisol & 272 & 1.36 & $<0.90-16.24$ & 874 & $<0.90$ & $<0.90-5.36$ & $<0.0001$ \\
\hline Corticosterone & 250 & 1.79 & $<0.90-28.55$ & 830 & 0.98 & $<0.90-24.45$ & $<0.0001$ \\
\hline 11-Deoxycortisol & 272 & 2.18 & $<0.90-19.59$ & 874 & 1.13 & $3.30-3.53$ & $<0.0001$ \\
\hline Androstenedione & 272 & 2.02 & $<0.90-29.87$ & 874 & $<0.90$ & $<0.90-6.27$ & $<0.0001$ \\
\hline 11-Deoxycorticosterone & 272 & $<0.90$ & $<0.90-1.55$ & 874 & $<0.90$ & $<0.90$ & $<0.0001$ \\
\hline \multirow[t]{2}{*}{ Testosterone } & M 149 & $<0.90$ & $<0.90-3.96$ & M 428 & $<0.90$ & $<0.90-2.63$ & 0.1651 \\
\hline & F 123 & $<0.90$ & $<0.90-1.54$ & F 446 & $<0.90$ & $<0.90-1.80$ & 0.0572 \\
\hline $17 \alpha$-Hydroxyprogesterone & 272 & 2.70 & $<0.90-59.72$ & 874 & $<0.90$ & $1.32-6.11$ & $<0.0001$ \\
\hline Progesterone & 272 & 4.29 & $<0.90-29.19$ & 874 & 4.87 & $<0.90-30.41$ & 0.0703 \\
\hline $\begin{array}{l}\text { (Androstenedione+17 } \alpha \text {-hydroxyprogesterone)/ } \\
\text { cortisol ratio }\end{array}$ & 272 & 0.19 & $0.01-3.84$ & 874 & 0.11 & $0.01-0.78$ & $<0.0001$ \\
\hline
\end{tabular}

${ }^{*} P$ values were calculated using the Mann-Whitney $U$ test for nonparametric data.

A

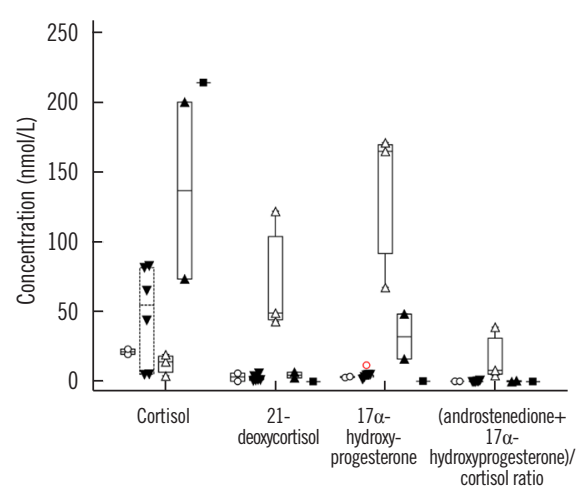

B

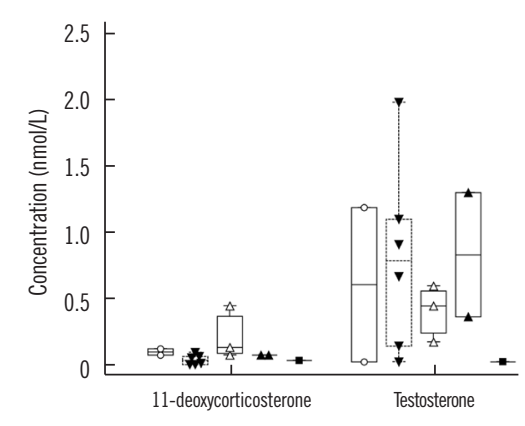

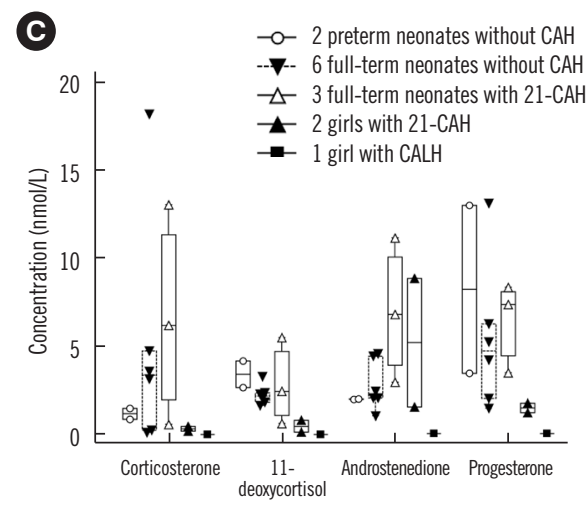

Fig. 2. Steroid profiles for 14 additional samples from neonates and children used to evaluate the clinical applicability of the LC-MS/MS method. Full-term neonates with 21-CAH $(\Delta)$ had distinctively low concentrations of cortisol, high concentrations of 21-deoxycortisol, 17 $\alpha$-hydroxyprogesterone, 11-deoxycorticosterone, and high values of (androstenedione+17 $\alpha$-hydroxyprogesterone)/cortisol ratio (A \& B). Other steroid hormone concentrations in full-term neonates with 21-CAH were variable (C). Two children with 21-CAH had high concentrations of cortisol and 17a-hydroxyprogesterone, progesterone, and androstenedione. Concentrations of other steroids were not distinctive. One child with CALH had high concentration of cortisol and low concentrations of other steroids.

Abbreviations: $\mathrm{CAH}$, congenital adrenal hyperplasia; 21-CAH, congenital adrenal hyperplasia caused by 21-hydroxylase deficiency; CALH, congenital adrenal lipoid hyperplasia due to the StAR mutation; LC-MS/MS, liquid chromatography-tandem mass spectrometry.

DBS samples from Korean neonates.

Intra-day precision and inter-day precision CVs were comparable with results in the literature and guidelines $[2,12,13,16$, 22]. Accuracy was within the acceptable range for neonatal screening using MS/MS [2, 4, 12, 13, 16, 17, 22]. Extraction recovery and the matrix effect were comparable to the previous findings and existing guidelines for neonatal screening by MS/ MS [2, 11-13, 16, 17, 20-23]. In our study, the bias between
LC-MS/MS and immunoassay findings changed over the measurement interval in a linear fashion, and there was a negative bias, with higher values measured by immunoassay than LCMS/MS over the entire range.

To avoid unnecessary tests, a sensitive and specific test, such as the LC-MS/MS method, is advantageous [1, 7]. Our method allows for simultaneous and rapid quantitation of nine steroids related to $\mathrm{CAH}$ with high specificity and without compromising 


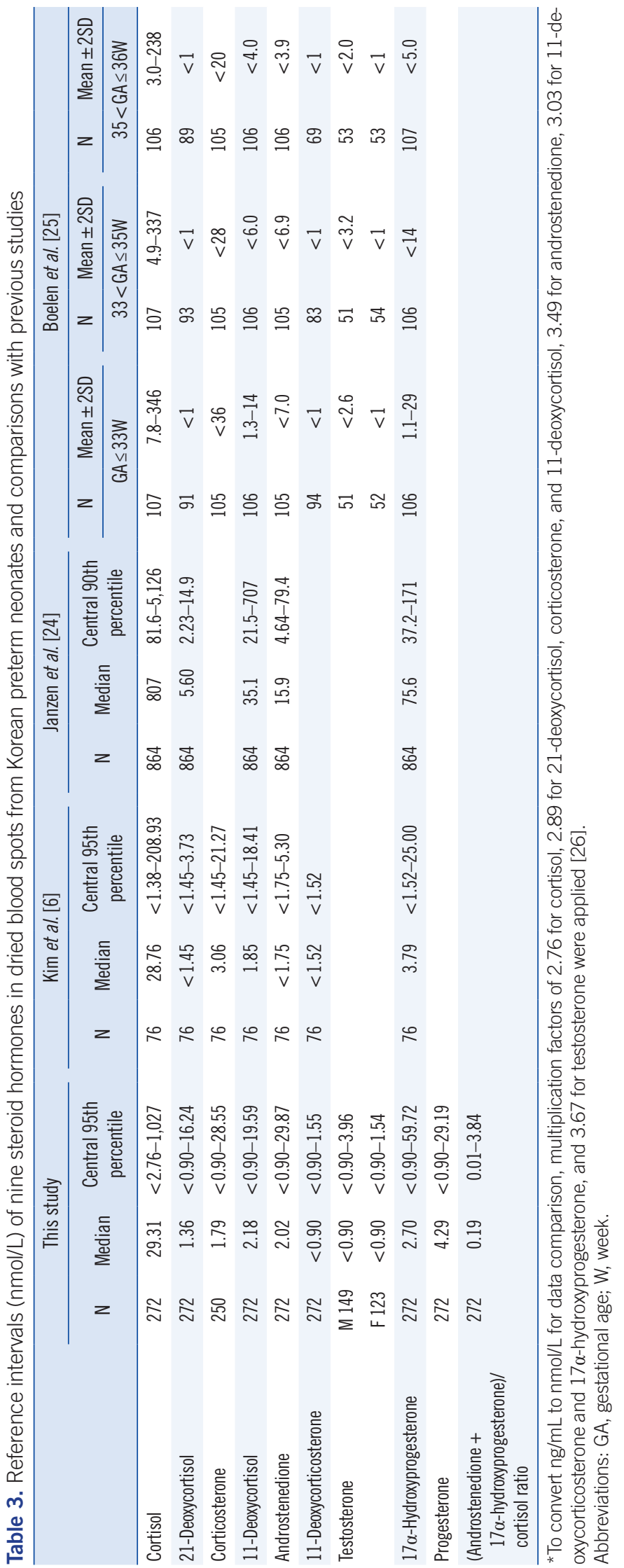

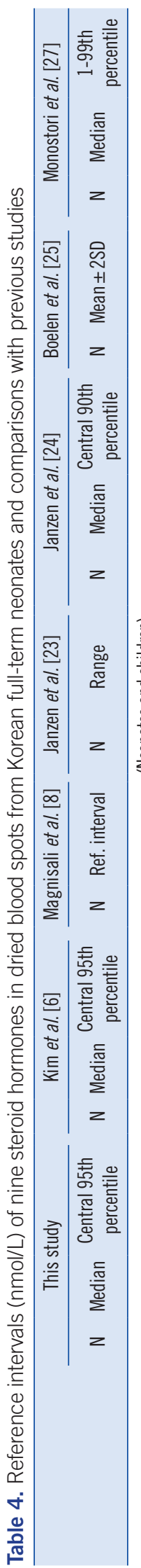

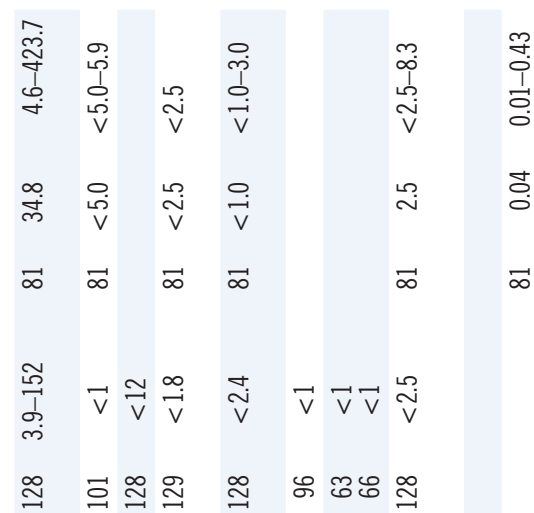

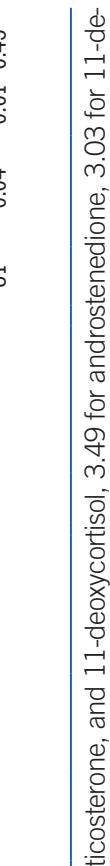

ని $\mathbb{2}$ ำ

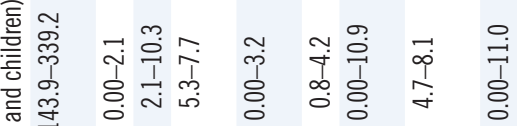
象

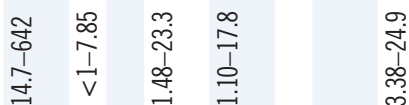

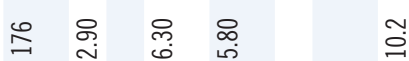

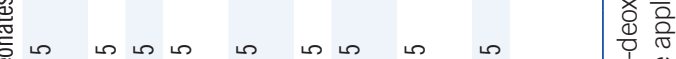

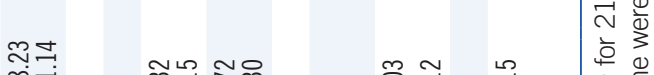

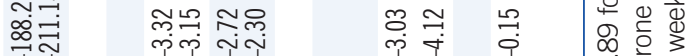

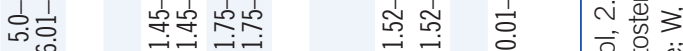

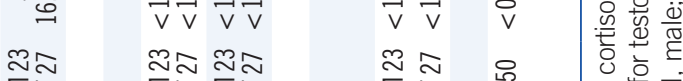

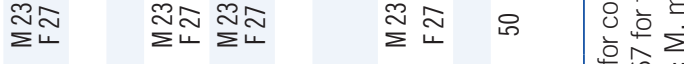

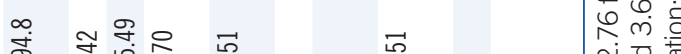

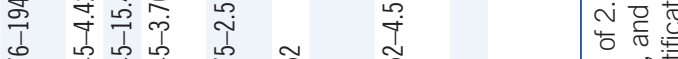

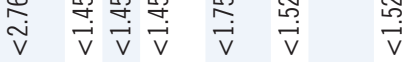

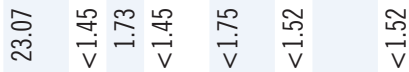

$\stackrel{\infty}{\wedge} \stackrel{\infty}{\wedge} \stackrel{\infty}{\wedge}$

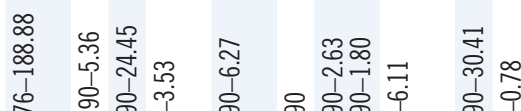

స

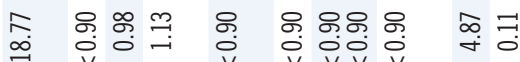

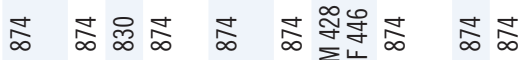

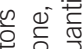

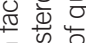

은

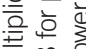

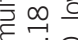

向 $\Phi^{-}$

离 就

5, 边

需

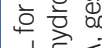

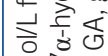

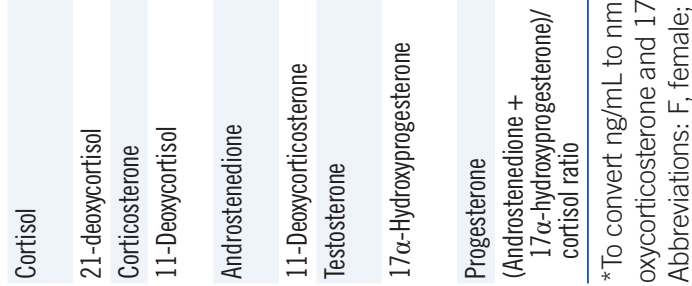


chromatographic resolution.

Differential diagnosis of CAH subtypes is possible by steroid profiling using the LC-MS/MS method [1]. In this method, diagnosis of $\mathrm{CAH}$ depends on not only the absolute quantification of $17 \alpha$-hydroxyprogesterone but also the ratio of multiple steroids to determine a positive result: 17 $\alpha$-hydroxyprogesterone (a direct substrate for 21-hydroxylase), cortisol (the final product of the adrenal enzyme's action), and androstenedione (which secondarily accumulates in 21-hydroxylase deficiency) [2]. Although the prevalence of $\mathrm{CAH}$ other than 21-hydroxylase deficiency is very low, and samples from patients with those diseases were not tested in this study, steroid profiling on the basis of ratios of multiple steroids, including precursor hormone and product hormones, used in this method has diagnostic strengths.

According to the CLSI guidelines [9], at least 120 samples for analysis are required to establish reference intervals. Some previous studies on the development and application of mass spectrometry for steroid profiling included less than 120 samples for establishing reference intervals $[6,8,23,25,27]$. Although the various reference intervals presented in these studies corresponded to different analytical conditions, they were quite similar to the present ones, as shown in Tables 3 and 4 [6, 8, 23$25,27]$. We included more than 120 samples for all nine steroids, especially testosterone and progesterone, which previous studies did not do [8, 25, 27].

Furthermore, we successfully demonstrated the clinical applicability of steroid profiling to differentiate between neonates with 21-hydroxylase deficiency and normal neonates. However, the reference interval was not established using samples from children to appropriately differentiate children with $\mathrm{CAH}$ from the normal ones. Considering that there was a 3-year-old girl with congenital adrenal lipoid hyperplasia due to StAR mutation whose cortisol concentration was higher than the upper limit of the reference interval for full-term neonates, whereas the other steroids were within the reference interval for full-term neonates, the reference interval of hormone analyses should be established separately for different age groups [6, 9].

The limitation of this study was the limited numbers of samples for validation, because the prevalence of CAH is low [25]. Previous studies also included limited numbers of $\mathrm{CAH}$ patients for clinical application due to the low prevalence of $\mathrm{CAH}$, but they reported successful clinical application of the methods in a manner similar to this study $[2,5,7]$.

In conclusion, our method produced lower values for $17 \alpha$ hydroxyprogesterone than an immunoassay, consistent with the relatively low analytical specificity of immunoassays compared with LC-MS/MS. We also established reference intervals for nine steroid hormones in Korean neonates, which were comparable with those in other populations. Steroid profiling by LC-MS/MS highlights the utility of secondary diagnostic markers as a means of reducing false-positive results due to stress- or illnessinduced transient elevation of $17 \alpha$-hydroxyprogesterone. Thus, our sensitive, specific, and accurate steroid profiling method is suitable for routine neonatal screening for $\mathrm{CAH}$.

\section{Authors' Disclosures of Potential Conflicts of Interest}

The authors declare that they have no conflicts of interest.

\section{Acknowledgements}

This study was supported by a grant from the Korea Health Technology R\&D Project, Ministry of Health \& Welfare, Korea (A120030).

\section{REFERENCES}

1. Rauh M. Steroid measurement with LC-MS/MS in pediatric endocrinology. Mol Cell Endocrinol 2009;301:272-81.

2. Rossi C, Calton L, Hammond G, Brown HA, Wallace AM, Sacchetta P, et al. Serum steroid profiling for congenital adrenal hyperplasia using liquid chromatography-tandem mass spectrometry. Clin Chim Acta 2010; 411:222-8.

3. Janzen N, Sander S, Terhardt M, Steuerwald U, Peter M, Das AM, et al. Rapid steroid hormone quantification for congenital adrenal hyperplasia (CAH) in dried blood spots using UPLC liquid chromatography-tandem mass spectrometry. Steroids 2011;76:1437-42.

4. Centers for Disease Control and Prevention. NSQAP: Newborn Screening Quality Assurance Program. Laboratory Quality Assurance and Standardization Programs. https://www.cdc.gov/labstandards/nsqap. html (Updated on Sep 2018).

5. Travers S, Martinerie L, Bouvattier $C$, Boileau P, Lombès M, Pussard E. Multiplexed steroid profiling of gluco- and mineralocorticoids pathways using a liquid chromatography tandem mass spectrometry method. J Steroid Biochem Mol Biol 2017;165:202-11.

6. Kim B, Lee MN, Park HD, Kim JW, Chang YS, Park WS, et al. Dried blood spot testing for seven steroids using liquid chromatography-tandem mass spectrometry with reference interval determination in the Korean population. Ann Lab Med 2015;35:578-85.

7. Seo JY, Park HD, Kim JW, Oh HJ, Yang JS, Chang YS, et al. Steroid profiling for congenital adrenal hyperplasia by tandem mass spectrometry as a second-tier test reduces follow-up burdens in a tertiary care hospital: a retrospective and prospective evaluation. J Perinat Med 2014;42: 121-7.

8. Magnisali P, Chalioti MB, Livadara T, Mataragas M, Paliatsiou S, Malamitsi-Puchner A, et al. Simultaneous quantification of $17 \alpha-\mathrm{OH}$ progesterone, 11-deoxycortisol, $\triangle 4$-androstenedione, cortisol and cortisone in newborn blood spots using liquid chromatography-tandem mass spec- 
trometry. J Chromatogr B Analyt Technol Biomed Life Sci 2011; 879:1565-72.

9. Horowitz GL, Altaie S, Boyd JC. Defining, establishing, and verifying reference intervals in the clinical laboratory, approved guideline. EP28-A3. 3rd ed. Wayne, PA: Clinical and Laboratory Standards Institute. 2010.

10. International Conference on Harmonisation (ICH). ICH topic Q2 (R1) Validation of analytical procedures: text and methodology. Int Conf Harmon 2005;1994:17.

11. Lacey JM, Minutti CZ, Magera MJ, Tauscher AL, Casetta B, McCann M, et al. Improved specificity of newborn screening for congenital adrenal hyperplasia by second-tier steroid profiling using tandem mass spectrometry. Clin Chem 2004;50:621-5.

12. U.S. Department of Health and Human Services Food and Drug Administration Center for Drug Evaluation and Research (CDER). BioanaIytical method validation guidance for industry 2018. https://www.fda. gov/downloads/drugs/guidances/ucm070107.pdf (Updated on Sep 2018).

13. European Medicines Agency Committee for Medicinal Products for Human Use (CHMP), 2011. Guideline on bioanalytical method validation. http://www.ema.europa.eu/docs/en_GB/document_library/Scientific_ guideline/2011/08/WC500109686.pdf (Updated on Sep 2018).

14. CLSI. Evaluation of the linearity of quantitative measurement procedures: A statistical approach; approved guideline. CLSI document EP06A. Wayne, PA: Clinical and Laboratory Standards Institute; 2003.

15. CLSI. Spectrometry for androgen and estrogen measurements in serum. CLSI guideline C57. 1st ed. Wayne, PA: Clinical and Laboratory Standards Institute, 2015.

16. CLSI. Screening by tandem mass spectrometry. CLSI guideline NBSO4. 2nd ed. Wayne, PA: Clinical and Laboratory Standards Institute; 2017.

17. CLSI. Measurement procedure comparison and bias estimation using patient samples. CLSI guideline EPO9c. 3rd ed. Wayne, PA: Clinical and Laboratory Standards Institute. 2018.

18. Dietzen DJ, Bennett MJ, Lo SF, Grey VL, Jones PM. Dried blood spot reference intervals for steroids and amino acids in a neonatal cohort of the National Children's Study. Clin Chem 2016;62:1658-67.

19. Ministry of Food and Drug Safety. Republic of Korea. Guideline on Bioanalytical Method Validation 2013;12. http://www.nifds.go.kr/brd/m_15/ view.do?seq=7018 (Updated on Sep 2018).

20. Matuszewski BK, Constanzer ML, Chavez-Eng CM. Strategies for the assessment of matrix effect in quantitative bioanalytical methods based on HPLC-MS/MS. Anal Chem 2003;75:3019-30.

21. Hicks RA, Yee JK, Mao CS, Graham S, Kharrazi M, Lorey F, et al. Precursor-to-product ratios reflect biochemical phenotype in congenital adrenal hyperplasia. Metabolomics 2014;10:123-31.

22. IUPAC. IUPAC Compendium of Chemical Terminology (The "Gold Book"). version 2.3.3. 2014. http://goldbook.iupac.org/ (Updated on Nov 2018).

23. Janzen N, Sander S, Terhardt M, Peter M, Sander J. Fast and direct quantification of adrenal steroids by tandem mass spectrometry in serum and dried blood spots. J Chromatogr B Analyt Technol Biomed Life Sci 2008;861:117-22.

24. Janzen N, Peter M, Sander S, Steuerwald U, Terhardt M, Holtkamp U, et al. Newborn screening for congenital adrenal hyperplasia: additional steroid profile using liquid chromatography-tandem mass spectrometry. J Clin Endocrinol Metab 2007;92:2581-9.

25. Boelen A, Ruiter AF, Claahsen-van der Grinten HL, Endert E, Ackermans MT. Determination of a steroid profile in heel prick blood using LC-MS/MS. Bioanalysis 2016;8:375-84.

26. Fiet J, Le Bouc Y, Guéchot J, Hélin N, Maubert MA, Farabos D, et al. A liquid chromatography/tandem mass spectometry profile of 16 serum steroids, including 21-deoxycortisol and 21-deoxycorticosterone, for management of congenital adrenal hyperplasia. J Endocr Soc 2017;1: 186-201.

27. Monostori P, Szabó P, Marginean O, Bereczki C, Karg E. Concurrent confirmation and differential diagnosis of congenital adrenal hyperplasia from dried blood spots: application of a second-tier LC-MS/MS assay in a cross-border cooperation for newborn screening. Horm Res Paediatr 2015;84:311-8. 